\title{
Segurança, reprodutibilidade, fatores intervenientes e aplicabilidade de testes de 1-RM
}

\author{
Raphael Mendes Ritti Dias ${ }^{1,2}$ \\ Ademar Avelar ${ }^{1}$ \\ Annelise Lins Menêses ${ }^{2}$ \\ Emanuel Péricles Salvador ${ }^{1}$ \\ Danilo Rodrigues Pereira da Silva ${ }^{1}$ \\ Edilson Serpeloni Cyrino \\ ${ }^{1}$ Grupo de Estudo e Pesquisa em Metabolismo, Nutrição e Exercício. Centro de Educação Física \\ e Esporte. Universidade Estadual de Londrina. Londrina, PR, Brasil. \\ ${ }^{2}$ Grupo de Pesquisa em Estilos de Vida e Saúde. Escola Superior de Educação Física. \\ Universidade de Pernambuco. Recife, PE, Brasil.
}

\begin{abstract}
Resumo: Um dos métodos mais utilizados para mensuração da força muscular é o teste de uma repetição máxima (1-RM), tendo em vista a sua versatilidade para aplicação em diferentes exercícios, a especificidade do movimento e o baixo custo operacional. Neste trabalho discutimos as evidências disponíveis a respeito da segurança, da reprodutibilidade, dos fatores intervenientes e da aplicabilidade prática do teste de 1-RM. Com base nas informações disponíveis até o presente momento, o teste de 1-RM parece ser um método seguro do ponto de vista ortopédico e cardiovascular e a sua reprodutibilidade depende, fundamentalmente, da realização de procedimentos de testagem adequados para a estabilização da carga, assim como do controle dos fatores intervenientes os quais podem influenciar no desempenho do teste. Embora a aplicabilidade para o diagnóstico e acompanhamento da força muscular seja ampla, a utilização de testes de 1-RM para a prescrição de treinamento com pesos ainda é bastante discutível.
\end{abstract}

Palavras-chave: Força muscular. Desempenho motor. Testes motores.

Safety, reliability, intervenient factors and applicability of 1-RM tests

\begin{abstract}
One of the most used methods for measuring muscular strength is the one repetition maximum test (1-RM), since its versatility for use in different exercises, its specificity of movement and low operating cost. Despite the advantages, some questions remain unclear. In this study we discuss about the available evidences regarding safety, reproducibility, intervenient factors and the practical applicability of the 1-RM test. The available evidence suggests that the 1-RM test is cardiovascular and orthopedic safely and Its reliability depends on the appropriate test procedures used for load stabilization as well as the control of intervenient factors that may influence the test performance. Although the applicability for the diagnosis and monitoring of muscular strength is large, the use of the 1-RM tests for the prescription of weight training is highly debatable.
\end{abstract}

Keywords: Muscular strength. Motor performance. Motor tests.

\section{Introdução}

Tanto 0 diagnóstico quanto 0 acompanhamento dos níveis de força muscular ao longo do tempo podem proporcionar valiosas informações para profissionais e pesquisadores que atuam com o desempenho esportivo e/ou com a saúde. Tais informações podem subsidiar a avaliação da capacidade funcional e da efetividade ou não de programas de exercícios físicos de diferentes naturezas, bem como favorecer o estabelecimento da sobrecarga de treinamento em programas direcionados à prevenção, reabilitação ou promoção da saúde ou, ainda, melhoria do desempenho atlético.
Nesse sentido, um dos métodos mais utilizados para análise da força muscular é o teste de uma repetição máxima (1-RM) (BALADY et al., 2000). Apesar de se tratar de um método que permite a avaliação indireta da força muscular, a partir da carga máxima levantada em exercícios com pesos livres ou máquinas, os escores alcançados guardam estreita relação com a capacidade de geração de força máxima de um determinado grupo muscular (VERDIJK et al., 2009). Desse modo, o teste de 1-RM tem ampla aceitação por parte da comunidade científica internacional. 
Tradicionalmente o teste de 1-RM é aplicado mediante tentativa e erro. Para tanto, em um ou mais exercícios escolhidos arbitrariamente são aplicadas cargas progressivas até que se atinja a carga máxima, ou seja, aquela que o avaliado consiga vencer a resistência oferecida em não mais do que uma única ação voluntária máxima, realizada a partir de um padrão pré-determinado de execução, respeitando-se o percurso completo do movimento, nas fases concêntrica e excêntrica. Considerando que a resistência oferecida na maioria das vezes não é suficientemente intensa para que se atinja a carga máxima em uma única tentativa, o Colégio Americano de Medicina do Esporte (ACSM, 2006) sugere que sejam adotadas de três a cinco tentativas por exercício, para o estabelecimento da força máxima. Vale destacar que, em cada uma das tentativas, 0 avaliado deve ser encorajado a tentar executar duas ações voluntárias máximas (2-RM) até que essa tarefa se torne impossível, momento no qual se configura a finalização do teste pelo alcance de 1RM.

Partindo-se do pressuposto de que para se atingir o máximo desempenho em uma tarefa motora repetitiva é necessário que o indivíduo esteja plenamente recuperado do esforço físico realizado anteriormente, a utilização de diversas tentativas e/ou a oferta de intervalos de recuperação demasiadamente curtos pode comprometer a qualidade das medidas produzidas em testes de 1-RM. Assim, assumindo-se que o tempo despendido para a execução de duas ações voluntárias máximas não é superior a poucos segundos (5-10 segundos aproximadamente), o sistema energético predominante é o sistema dos fosfagênios (ATP-CP). Informações disponíveis na literatura têm indicado que a plena recuperação dessa via metabólica é alcançada em intervalos de três a cinco minutos, intervalo de tempo que vem sendo sugerido e adotado por diferentes pesquisadores entre as tentativas utilizadas em testes de 1-RM (CRONIN; HENDERSON, 2004; GURJÃO et al., 2005; NASCIMENTO et al. 2012; RITTI-DIAS et al., 2005, 2009, 2011; SILVA-BATISTA et al., 2011; SOARES-CALDEIRA et al., 2009; TIGGEMANN et al., 2011), embora não exista consenso a esse respeito na literatura (BENTON et al., 2009; LEVINGER et al., 2009; PHILLIPS et al., 2009; PLOUTZ-SNYDER; GIAMIS et al., 2001).
Com base nessas informações, a utilização do teste de 1-RM de maneira adequada pode apresentar algumas vantagens em relação a outros métodos empregados para a avaliação da força muscular, a saber: 1) os testes podem ser aplicados em diferentes exercícios para análise da força máxima de diferentes grupos musculares; 2) a especificidade do movimento é garantida em indivíduos a serem submetidos a treinamento com pesos livres/máquinas ou naqueles que já vêm sendo exercitados com esse tipo de treinamento; 3) não existe necessidade da aquisição de equipamentos sofisticados e de alto custo operacional para a sua aplicação.

Entretanto, existe ainda muita controvérsia e uma certa insegurança com relação à utilização de testes de 1-RM no campo da pesquisa e, principalmente, nos diferentes ambientes de atuação profissional (academias, clubes e clínicas). Assim, procuraremos discutir neste trabalho importantes questões relacionadas à utilização de testes de 1-RM para análise da força muscular, tais como a segurança, a reprodutibilidade, os fatores intervenientes, a aplicação e recomendações práticas em diversas populações, com base nas informações disponíveis na literatura até o mês de fevereiro de 2013, de acordo com as bases de dados Pubmed, Scielo e Lilacs, nas referências utilizadas nos artigos encontrados e na experiência dos estudos conduzidos pelo nosso laboratório. As palavraschave utilizadas para a busca das informações foram: familiarização, reprodutibilidade, uma repetição máxima, teste de 1-RM, força muscular, força máxima, exercício com pesos, exercício resistido, exercício de força e suas respectivas traduções para a língua inglesa.

\section{Riscos de lesão ou dor tardia causados por testes de 1-RM}

Embora o teste de 1-RM seja um dos testes mais freqüentemente citados na literatura, apenas um estudo se preocupou em analisar especificamente a incidência de lesões em testes de 1-RM (POLLOCK et al., 1991). Nesse estudo, 70 idosos foram submetidos a testes de 1-RM nos exercícios extensão de joelhos e supino inclinado. Os resultados indicaram 11 lesões (cinco no joelho, cinco no ombro ou braço e uma nas costas), dentre as quais sete teriam sido acarretadas exclusivamente pelo teste de 1-RM, ao passo que as demais ocorreram, possivelmente, em virtude de lesões prévias. 
Dos demais trabalhos que utilizaram o teste de 1-RM em suas investigações, poucos estudos relataram a incidência de lesões em decorrência do teste de 1-RM. Nichols et al. (1993), ao submeterem idosos ao teste de 1-RM nos exercícios desenvolvimento, supino em banco horizontal, puxada na polia alta, remada e extensão de joelhos, reportaram que apenas $2,8 \%$ dos avaliados se lesionaram durante o teste. De forma semelhante, Shaw et al. (1995) relataram a incidência de lesões em $2,4 \%$ dos sujeitos sem experiência prévia em exercícios com pesos quando submetidos a testes de 1-RM nos exercícios supino em banco horizontal, rosca direta de bíceps, extensão de joelhos e panturrilha. Por outro lado, nenhuma lesão foi identificada em indivíduos com experiência prévia em exercícios com pesos.

Não se pode desprezar que a escassez de informações sobre a incidência de lesões no teste de 1-RM pode estar relacionada à omissão, por parte dos pesquisadores, de informações relacionadas a possíveis eventos adversos ocorridos em decorrência deste procedimento. Entretanto, a experiência do nosso laboratório com a aplicação de testes de 1-RM, em diferentes populações (GURJÃO et al., 2005; NASCIMENTO et al., 2012; RITTI-DIAS et al., 2005, 2009, 2011; SALVADOR et al., 2005; SOARES-CALDEIRA et al., 2009), nos permite afirmar que o risco de lesões associadas a esses testes é mínimo quando os procedimentos de aplicação seguem o rigor metodológico necessário (processo de familiarização aos exercícios e ao teste propriamente dito, supervisão adequada, intervalos de recuperação prolongados entre as tentativas e os testes, repetição dos testes somente em dias não consecutivos). Vale destacar que até o momento não identificamos, nos sujeitos dos nossos estudos, nenhum tipo de lesão que pudesse ser atribuída a testes de 1-RM.

Adicionalmente, alguns estudos têm analisado a ocorrência de dor tardia após testes de 1-RM (BARNARD et al., 1999; KAELIN et al., 1999; SHAW et al., 1995). Os resultados encontrados até o momento têm indicado que a magnitude da dor tardia após testes de 1-RM é relativamente baixa (variando de 1 a 2 pontos em uma escala de 4 pontos). Vale ressaltar que grande parte das informações disponíveis a esse respeito tem sido obtida em amostras compostas por sujeitos idosos e/ou sem experiência prévia em treinamento de força, ou seja, em populações que podem ser consideradas de maior risco, o que fortalece os indicativos de que a aplicação de testes de 1-RM parece bastante segura para análise da força muscular em diferentes populações.

Apesar dos relatos de que a ocorrência de dor tardia é um evento relativamente freqüente, principalmente em indivíduos não-treinados quando submetidos a exercícios de força, os resultados encontrados em testes de 1-RM não parecem diferir daqueles observados em outros testes utilizados para a avaliação da força muscular. Entretanto, existe necessidade do desenvolvimento de estudos bem controlados que testem essa hipótese para uma resposta definitiva.

\section{Segurança cardiovascular do teste de 1-RM}

Entre os estudos que investigaram respostas da pressão arterial (PA) durante a realização de testes de 1-RM, há que se destacar um estudo conduzido com atletas de culturismo, praticantes recreativos de treinamento de força e iniciantes (FLECK; DEAN, 1987). Ao analisarem o comportamento da PA por meio de medidas obtidas pelo método intra-arterial, os pesquisadores encontraram aumentos na ordem de $41 \%$ a $52 \%$ na PA sistólica e de $39 \%$ a $95 \%$ na PA diastólica dos três grupos investigados, nos exercícios de extensão de joelhos e desenvolvimento, respectivamente. Incrementos na ordem de $91 \%$ na PA média já haviam sido descritos anteriormente durante testes de 1-RM no exercício leg press, em jovens saudáveis (MACDOUGALL et al., 1985).

Em idosos, as respostas observadas na PA sistólica e diastólica durante testes de 1-RM foram de maior magnitude no leg press do que nos exercícios de flexão de joelhos e extensão de joelhos (DE VOS et al., 2008). Tais resultados, obtidos por meio de pletismografia, sugerem que os exercícios que envolvem maior massa muscular promovem maiores incrementos na PA. Adicionalmente, a elevação da PA durante o exercício extensão de joelhos realizado com $40 \%$, $80 \%$ e $100 \%$ de 1 -RM até a fadiga parece ser semelhante (25-28\%), em hipertensos monitorizados pelo método intra-arterial (GOMIDES et al., 2010).

Com relação à ocorrência de arritmias, contrações atriais prematuras foram observadas 
durante testes de 1-RM em apenas um indivíduo participante de um programa de reabilitação cardíaca (KAELIN et al., 1999). Todavia, de acordo com os autores, essas contrações já existiam em condições basais. Adicionalmente, uma incidência de movimentos anormais na parede ventricular durante a realização de testes de 1-RM, na ordem de $6,7 \%$ e $4,8 \%$, foi constatada, por meio de ecocardiograma, nos exercícios extensão de joelhos e flexão de cotovelos, respectivamente, em idosos com disfunção ventricular esquerda (WERBER-ZION et al., 2004). Essas alterações foram semelhantes às observadas em teste máximo em esteira $(4,8 \%)$. Apesar disso, os pesquisadores que conduziram esses dois estudos afirmam que as respostas arrítmicas encontradas durante os testes de 1-RM não representam risco cardiovascular.

Em suma, embora os resultados dos estudos revisados tenham indicado elevação da PA durante testes de 1-RM, às respostas cardiovasculares encontradas foram consideradas normais, confirmando a segurança cardiovascular da aplicação de testes de 1-RM em indivíduos jovens, idosos, hipertensos e normotensos. Além disso, as arritmias verificadas durante testes de 1RM podem ser consideradas dentro dos padrões de normalidade frente ao esforço físico, demonstrando mais uma vez a segurança desse tipo de teste, inclusive, para cardiopatas, particularmente, aqueles com disfunção ventricular esquerda.

\section{Confiabilidade de testes de 1-RM}

A qualidade das informações produzidas por testes de 1-RM tem sido alvo de inúmeras investigações. O primeiro estudo bem controlado nessa direção foi conduzido em mulheres jovens e idosas (PLOUTZ-SNYDER; GIAMIS, 2001). Embora nessa investigação os pesquisadores tenham encontrado um alto coeficiente de reprodutibilidade $(r=0,94)$, aumentos significantes na força máxima foram verificados entre as diferentes sessões de testes de 1-RM, sem que houvesse qualquer tipo de intervenção ao longo do período experimental. O platô da força máxima foi atingido somente entre a terceira/quarta sessão em mulheres jovens e entre a oitava/nona sessão em mulheres idosas, destacando a importância da aplicação de múltiplos testes para a familiarização dos sujeitos avaliados e, consequentemente, para a geração de uma medida confiável de força máxima. Obviamente, esses achados colocaram em dúvida a precisão e a confiabilidade dos estudos anteriores que buscaram avaliar as modificações na força muscular após diferentes períodos de intervenção sem, contudo, o controle dessa importante variável. Portanto, na prática, grande parte dos ganhos de força encontrados poderia ser atribuído, simplesmente, ao processo de familiarização com os testes de 1-RM e não à efetividade da intervenção.

Posteriormente, novas investigações indicaram que o número de sessões necessárias para familiarização ao teste de 1-RM parece ser diferente em crianças (GURJÃO et al., 2005), adultos jovens (BENTON et al., 2009; CRONIN; HENDERSON, 2004; RITTI-DIAS et al., 2005, 2009, 2011; SOARES-CALDEIRA et al., 2009; SILVA-BATISTA et al., 2011; TIGGEMANN et al., 2011), adultos de meia-idade (LEVINGER et al., 2009) e idosos (NASCIMENTO et al., 2012;

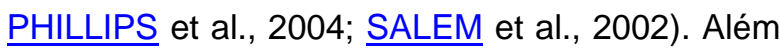
disso, em exercícios de maior complexidade (multiarticulares) parece existir a necessidade de um maior número de sessões de familiarização aos testes de 1-RM quando comparados a exercícios de menor complexidade ( $\underline{\mathrm{CRONIN}}$; HENDERSON, 2004; RITTI-DIAS et al., 2005).

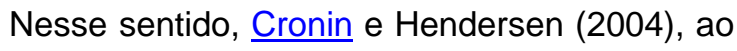
analisarem a reprodutibilidade de testes de 1-RM em indivíduos jovens submetidos aos exercícios supino em banco horizontal, agachamento unilateral e agachamento bilateral, encontraram um aumento significante na força máxima entre a primeira e a segunda sessão de testes, em todos os exercícios analisados. Todavia, diferente do observado no exercício supino em banco horizontal, nos exercícios agachamento unilateral e agachamento bilateral os valores de força máxima aumentaram até a terceira sessão, o que sugere que exercícios mais complexos demandariam maior tempo para a estabilização da carga de 1-RM. Resultados semelhantes foram encontrados em estudo do nosso laboratório (RITTI-DIAS et al., 2005), cujo tempo para familiarização no exercício rosca direta foi menor em comparação a exercícios mais complexos (supino em banco horizontal e agachamento). 
Nesses dois estudos, a estabilização das cargas para todos os exercícios ocorreu em até três sessões de familiarização.

Adicionalmente, Benton et al. (2009), ao submeterem mulheres não-treinadas em exercícios de força a testes de 1-RM em três dias não consecutivos, observaram melhoria no desempenho a cada testagem no exercício leg press, ao passo que no exercício supino em banco horizontal só foram identificados incrementos significantes na força nas comparações entre a primeira e a última sessão de testagem.

Recentemente, outro estudo conduzido pelo nosso laboratório (RITTI-DIAS et al., 2011) indicou que homens jovens, não-experientes com o treinamento de força, parecem necessitar de duas a três sessões de testes de 1-RM para a estabilização da carga, enquanto seus pares com experiência prévia de pelo menos 24 meses neste tipo de treinamento o processo de familiarização a testes de 1-RM parece desnecessário.

Com base nas informações apresentadas, a quantidade necessária de sessões de familiarização a testes de 1-RM para se obter um registro confiável da força máxima parece ser dependente da faixa etária, do sexo, do tipo de exercício e do nível de experiência prévia do avaliado com a prática de exercícios de força. Vale destacar que a experiência do nosso laboratório em tratar informações obtidas em múltiplos testes de 1-RM, em diferentes populações, tem demonstrado que a análise do comportamento dos avaliados em testes de 1-RM, a partir de procedimentos estatísticos relativamente simples, como a aplicação de coeficiente intra-classe, não parece ser suficientemente sensível para garantir a qualidade ou não das informações produzidas em testes repetitivos. Assim, a incorporação de procedimentos estatísticos mais robustos e que possibilitem comparações múltiplas em combinação com análise de concordância individual entre as medidas obtidas em diferentes sessões de testes de 1-RM é indispensável para a determinação do momento de estabilização das cargas e, conseqüentemente, de um registro mais fidedigno dos valores de força máxima.
As principais informações relatadas nos estudos que investigaram a necessidade ou não da aplicação de sessões de familiarização a testes de 1-RM são apresentadas na tabela 1.

\section{Fatores intervenientes sobre o desempenho motor em testes de 1-RM}

Há evidências de que a realização de alongamento (passivo e/ou ativo) para extensores e/ou flexores de joelho previamente a testagem pode reduzir em cerca de 4 a $13 \%$ o desempenho em testes de 1-RM, em exercícios que exigem movimentos de extensão e flexão de joelho (KOKKONEN et al., 1998; NELSON et al., 2001, 2005; TRICOLI; PAULO, 2002). Em contrapartida, a realização de aquecimento específico ou facilitação neuromuscular proprioceptiva previamente a execução de testes de 1-RM parece não afetar o desempenho no exercício

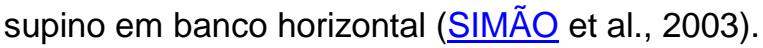

Outra variável que pode afetar o desempenho em testes de 1-RM é o intervalo de recuperação utilizado entre as tentativas. Weir et al. (1994), ao analisarem o efeito de um, três, cinco e 10 minutos de intervalo entre as tentativas no exercício supino em banco horizontal, observaram que aproximadamente $25 \%$ dos indivíduos testados não conseguiram repetir o desempenho em uma segunda tentativa quando o intervalo adotado foi de um minuto, ao passo que com três minutos de intervalo nenhuma queda de desempenho foi identificada. De forma similar, Matuszak et al. (2003), ao investigarem o efeito de diferentes intervalos (um, três e cinco minutos) entre as tentativas, no exercício agachamento, verificaram que o intervalo de um minuto resultou em $23,5 \%$ de falhas na tentativa subseqüente, ao passo que o intervalo de três minutos resultou em apenas $5,9 \%$ de falhas. Esses resultados sugerem que um intervalo de no mínimo três minutos deve ser utilizado entre as diferentes tentativas em testes de 1-RM.

Com relação ao impacto da utilização de algumas substâncias ergogênicas, como a cafeína, para a melhoria do desempenho em testes de 1-RM os resultados preliminares são inconclusivos. Em estudo duplo-cego, placebo controlado, Beck et al. (2006) veificaram que a ingestão de cafeína $(2,1-3,0 \mathrm{mg} / \mathrm{kg})$ resultou em aumento significante $(2,3 \%)$ na força máxima no 
exercício supino em banco horizontal, embora o mesmo efeito não tenha sido identificado no exercício extensão de joelhos, o que foi confirmado posteriormente por outros pesquisadores (ASTORINO et al., 2008).

O desempenho no teste de 1-RM também parece ser afetado pela temperatura ambiente e pela desidratação. Em estudo realizado por Hedley et al. (2002) foi verificado que a realização de testes de 1-RM sob temperatura ambiente em torno de $41^{\circ}$ reduziu a força máxima em aproximadamente $3 \%$ no exercício leg press, em comparação ao desempenho observado em condições normais (22-24ํ).

Schoffstall et al. (2001), por sua vez, analisaram a influência da hidratação sobre o desempenho em testes de 1-RM no exercício supino em banco horizontal. O protocolo utilizado consistiu da exposição dos sujeitos a duas horas de sauna, seguidas ou não de um processo de reidratação. Os resultados indicaram que 0 desempenho foi reduzido em torno de $5 \%$ nos indivíduos que não foram submetidos à reidratação após a sauna.

Em síntese, as informações até o presente momento sugerem que a execução de exercícios de alongamento imediatamente antes da testagem, a adoção de curtos intervalos de recuperação entre as tentativas (<3 minutos), ambiente quente e desidratação podem prejudicar o desempenho em testes de 1-RM. Entretanto, pouco se sabe sobre a possível influência de outras variáveis como o número máximo de tentativas a serem utilizadas, o período do dia em que esses testes são executados, o uso de ergogênicos nutricionais, entre outras.

Tabela 1. Estudos sobre familiarização a testes de 1-RM.

\begin{tabular}{|c|c|c|c|c|c|c|c|}
\hline Estudo & $\begin{array}{c}n \\
\text { (sexo) }\end{array}$ & $\begin{array}{l}\text { Idade } \\
\text { (anos) }\end{array}$ & $\begin{array}{c}\text { № de } \\
\text { exercícios }\end{array}$ & $\begin{array}{c}\text { № de } \\
\text { tentativas }\end{array}$ & $\begin{array}{c}\text { № de } \\
\text { sessões }\end{array}$ & Variação & Resultados \\
\hline Benton et al. (2009) & $19(\mathrm{M})$ & $35,5 \pm 2,1$ & 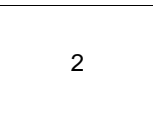 & $3-5$ & 3 & $\begin{array}{l}\text { SUP }(6,0 \%) \\
\operatorname{LP}(14,4 \%)\end{array}$ & $\begin{array}{l}\text { Estabilização das cargas na } \\
\text { terceira sessão para o SUP } \\
\text { e necessidade de mais } \\
\text { sessões para o LP. }\end{array}$ \\
\hline$\frac{\text { Cronin e Henderson }}{(2004)}$ & $10(\mathrm{H})$ & $21,0 \pm 2,7$ & 3 & 6 & 4 & $\begin{array}{l}\text { SUP }(13,6 \%) \\
\text { AGAu }(16,8 \%) \\
\text { AGAb }(15 \%)\end{array}$ & $\begin{array}{l}\text { Estabilização das cargas na } \\
\text { segunda (SUP) e terceira } \\
\text { (AGAu e AGAb) sessões. }\end{array}$ \\
\hline Gurião et al. (2005) & $9(\mathrm{H})$ & $9,5 \pm 0,5$ & 2 & 3 & 8 & $\begin{array}{l}\operatorname{ME}(30,2 \%) \\
\operatorname{RDB}(22,7 \%)\end{array}$ & $\begin{array}{c}\text { Estabilização das cargas na } \\
\text { terceira (ME) e quinta (RDB) } \\
\text { sessões. }\end{array}$ \\
\hline Levinger et al. (2009) & $\begin{array}{l}13(\mathrm{H}) \\
14(\mathrm{M})\end{array}$ & $50,7 \pm 1,3$ & 7 & $3-6$ & 3 & $\begin{array}{c}\text { SUP }(2,0 \%) \\
\text { RDB }(1,5 \%) \\
\text { LP }(4,3 \%) \\
\text { PF }(0,6 \%) \\
\text { TP }(2,5 \%) \\
\text { ME }(3,8 \%) \\
\text { RB }(1,1 \%)\end{array}$ & $\begin{array}{l}\text { Estabilização das cargas na } \\
\text { segunda sessão. }\end{array}$ \\
\hline$\frac{\text { Nascimento et al. }}{(2012)}$ & $45(\mathrm{M})$ & $65,5 \pm 4,3$ & 3 & 3 & 3 & $\begin{array}{l}\text { SUP }(4,2 \%) \\
\operatorname{LP}(11,7 \%) \\
\operatorname{RDB}(15 \%))\end{array}$ & $\begin{array}{l}\text { Estabilização das cargas } \\
\text { entre a segunda e terceira } \\
\text { sessões }\end{array}$ \\
\hline Phillips et al. (2004) & $\begin{array}{l}16(\mathrm{H}) \\
31(\mathrm{M})\end{array}$ & $\begin{array}{c}75,8 \pm 7,5 \\
75,2 \pm 17,3\end{array}$ & 2 & NR & 3 & $\begin{array}{c}\text { SUP }(H=5,9 \% ; M=2,9 \%) \\
\operatorname{LP}(H=2,3 \% ; M=3,9)\end{array}$ & $\begin{array}{l}\text { Estabilização das cargas na } \\
\text { terceira sessão }\end{array}$ \\
\hline$\frac{\text { Ploutz-Snyder e Giamis }}{(2001)}$ & $\begin{array}{l}7 \mathrm{~A}(\mathrm{M}) \\
6 \mathrm{I}(\mathrm{M})\end{array}$ & $\begin{array}{l}23,0 \pm 4,0 \\
66,0 \pm 5,0\end{array}$ & 1 & $3-8$ & $\begin{array}{l}2-5(\mathrm{~A}) \\
7-10(\mathrm{I})\end{array}$ & $\operatorname{ME}(A=11,8 \% ; I=20,8 \%)$ & $\begin{array}{c}\text { Estabilização das cargas } \\
\text { entre a terceira e quarta (A) } \\
\text { e entre a oitava e nona (I) } \\
\text { sessões. }\end{array}$ \\
\hline
\end{tabular}

Nota. $\mathrm{H}$ = homens; $\mathrm{M}=$ mulheres; SUP = Supino em banco horizontal; $\mathrm{LP}=$ Leg press; $\mathrm{AGA}=\mathrm{Agachamento}$; $\mathrm{AGAu}=$ Agachamento unilateral; $A G A b=$ Agachamento bilateral; $R D B=$ Rosca direta de bíceps; $P F=$ Puxada pela frente; TP = Tríceps na polia; $\mathrm{ME}=$ Mesa extensora; $\mathrm{RB}=$ Remada baixa; $\mathrm{MF}=$ Mesa flexora; $\mathrm{FL}=$ Flexão plantar de tornozelo; $\mathrm{NR}=$ Não relatado; $\mathrm{E}=$ Experientes; $\mathrm{NE}=$ Não-experientes; $\mathrm{SD}=$ Sedentários; FA = Fisicamente ativos; TF = Treinados em força; $\mathrm{A}$ = Adultas; I = Idosas. 
Tabela 1. (continuação).

\begin{tabular}{|c|c|c|c|c|c|c|c|}
\hline Estudo & n (sexo) & $\begin{array}{l}\text { Idade } \\
\text { (anos) }\end{array}$ & $\begin{array}{c}\text { № de } \\
\text { exercícios }\end{array}$ & $\begin{array}{c}\text { № de } \\
\text { tentativas }\end{array}$ & $\begin{array}{c}\text { № de } \\
\text { sessões }\end{array}$ & Variação & Resultados \\
\hline$\underline{\text { Ritti-Dias et al. (2005) }}$ & $21(H)$ & $24,5 \pm 3,8$ & (5) & 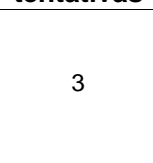 & (20000 & $\begin{array}{l}\text { SUP }(2,4 \%) \\
\text { AGA }(3,3 \%) \\
\operatorname{RDB}(5,5 \%)\end{array}$ & $\begin{array}{l}\text { Estabilização das } \\
\text { cargas na segunda } \\
\text { (RDB) e terceira } \\
\text { (SUP e AGA) } \\
\text { sessões. }\end{array}$ \\
\hline 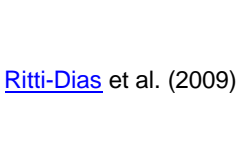 & $18(\mathrm{M})$ & $22,4 \pm 2,8$ & 3 & 3 & 5 & $\begin{array}{l}\text { SUP }(6,1 \%) \\
\text { AGA }(8,0 \%) \\
\operatorname{RDB}(12,6 \%)\end{array}$ & $\begin{array}{l}\text { Estabilização das } \\
\text { cargas na segunda } \\
\text { (AGA) e terceira } \\
\text { (SUP e RDB) } \\
\text { sessões. }\end{array}$ \\
\hline$\underline{\text { Ritti-Dias et al. (2011) }}$ & $\begin{array}{c}16 \mathrm{E}(\mathrm{H}) \\
14 \mathrm{NE}(\mathrm{H})\end{array}$ & 18 a 30 & 2 & 3 & 4 & $\begin{array}{l}\text { SUP }(E=1,2 \% ; N E=10,2 \%) \\
\text { AGA } \quad(E=3,3 \% ; N E=11,2 \%)\end{array}$ & $\begin{array}{c}\text { E: Sem diferença } \\
\text { entre as sessões; } \\
\text { NE: Estabilização } \\
\text { das cargas na } \\
\text { segunda (AGA) e } \\
\text { terceira (SUP) } \\
\text { sessões. }\end{array}$ \\
\hline$\underline{\text { Salem et al. (2002) }}$ & $\begin{array}{c}6(H) \\
24(M)\end{array}$ & $65,2 \pm 6,3$ & 4 & 3 & 4 & $\begin{array}{l}\operatorname{LP}(13,3 \%) \\
\operatorname{ME}(3,0 \%) \\
\operatorname{MF}(13,5 \%) \\
\operatorname{FP}(12,3 \%)\end{array}$ & $\begin{array}{l}\text { Estabilização das } \\
\text { cargas na } \\
\text { segunda sessão. }\end{array}$ \\
\hline$\frac{\text { Silva-Batista et al. }}{(2011)}$ & $\begin{array}{l}8(\mathrm{H}) \\
8(\mathrm{M})\end{array}$ & $\begin{array}{l}25,4 \pm 4,0 \\
20,4 \pm 1,7\end{array}$ & 1 & Até 5 & 5 & $\begin{array}{c}\text { AGA } \\
(H=18 \% ; M=15 \%)\end{array}$ & $\begin{array}{c}\text { Estabilização das } \\
\text { cargas na terceira } \\
(\mathrm{M}) \text { e quarta }(\mathrm{H}) \\
\text { sessões. }\end{array}$ \\
\hline$\frac{\text { Soares-Caldeira et al. }}{(2009)}$ & $27(\mathrm{M})$ & $21,6 \pm 2,5$ & 3 & 3 & 5 & $\begin{array}{c}\text { SUP }(6,0 \%) \\
\text { AGA }(5,4 \%) \\
\operatorname{RDB}(11,7 \%)\end{array}$ & $\begin{array}{l}\text { Estabilização das } \\
\text { cargas entre a } \\
\text { terceira e quarta } \\
\text { sessões }\end{array}$ \\
\hline$\frac{\text { Tiggemann et al. }}{(2011)}$ & $\begin{array}{l}10 \mathrm{SD}(\mathrm{M}) \\
10 \mathrm{FA}(\mathrm{M}) \\
10 \mathrm{TF}(\mathrm{M})\end{array}$ & 18 a 34 & 2 & Até 5 & 2 & $\begin{array}{l}\text { SUP (SD }=2,1 \% ; F A=0.9 ; T F=1,6 \%) \\
\operatorname{LP}(S D=3,2 \% ; F A=2,3 ; T F=4,6 \%)\end{array}$ & $\begin{array}{c}\text { Não houve } \\
\text { estabilização das } \\
\text { cargas em nenhum } \\
\text { dos três grupos } \\
\text { experimentais, em } \\
\text { ambos os } \\
\text { exercícios }\end{array}$ \\
\hline
\end{tabular}

Nota. $\mathrm{H}=$ homens; $\mathrm{M}=$ mulheres; SUP = Supino em banco horizontal; $\mathrm{LP}=$ Leg press; $\mathrm{AGA}=$ Agachamento; $\mathrm{AGAu}=$ Agachamento unilateral; $\mathrm{AGAb}=$ Agachamento bilateral; $\mathrm{RDB}=$ Rosca direta de bíceps; $\mathrm{PF}=$ Puxada pela frente; $\mathrm{TP}=$ Tríceps na polia; $\mathrm{ME}=$ Mesa extensora; $\mathrm{RB}=$ Remada baixa; $\mathrm{MF}=$ Mesa flexora; $\mathrm{FL}=$ Flexão plantar de tornozelo; $\mathrm{NR}=$ Não relatado; $\mathrm{E}=$ Experientes; $\mathrm{NE}=$ Não-experientes; $\mathrm{SD}=$ Sedentários; FA = Fisicamente ativos; TF = Treinados em força; $\mathrm{A}$ = Adultas; $\mathrm{I}$ = Idosas.

\section{Aplicabilidade de testes de 1-RM}

A utilização de testes de 1-RM para identificar alterações na força máxima após programas de intervenção é bastante comum e frequente em pesquisas científicas, uma vez que esse método apresenta importantes vantagens frente aos demais (dinamometria isocinética, plataforma de força, etc...), tais como: baixo custo financeiro, facilidade e versatilidade para a sua aplicação em diversos movimentos e especificidade em relação ao tipo de contração utilizada durante as sessões de treinamento.

Vale destacar que a validade do teste de 1-RM tem sido comprovada, independente do gênero e da faixa etária, a partir de comparações com dinamometria, método considerado padrão-ouro para avaliação da carga máxima (VERDIJK et al., 2009). Além disso, o teste de 1-RM tem apresentado boa sensibilidade (ABERNETHY; JURIMAE, 1996; MURPHY; WILSON, 1997), O que é fundamental para a análise das modificações na força muscular induzidas por programas de intervenção. Além disso, alguns estudos têm demonstrado a eficiência do teste de 1-RM para o diagnóstico do nível de aptidão física (SCHILLER et al., 2000) e de doenças crônicas (TAAFFE et al., 1995).

Por outro lado, a utilização dos resultados de testes de 1-RM para a prescrição de treinamento com pesos tem recebido inúmeras críticas ao longo dos últimos anos, sobretudo, pela grande variação no número de repetições atingidas em diferentes exercícios, a partir de cargas estabelecidas com base em percentuais de 1-RM (SIMÃO et al., 2006). De fato, os estudos que analisaram o número de repetições executadas com cargas relativas a 1-RM têm observado que 
não existe um padrão bem definido ( $\underline{\mathrm{CHAGAS}}$ et al., 2005; SALVADOR et al., 2005), visto que existe uma dependência do grupamento muscular envolvido, do sexo, do nível de treinamento, do número de séries e do intervalo de recuperação entre as séries, entre outros fatores.

Assim, a utilização das cargas relativas baseadas em resultados de testes 1-RM para a prescrição do treinamento de força produz uma falsa impressão de que o treinamento está sendo realizado com cargas submáximas, o que nem sempre é verdadeiro, visto que o conceito de exercício máximo está diretamente relacionado com a ocorrência de fadiga, ao passo que 0 exercício submáximo, via de regra, é interrompido antes da fadiga. Portanto, se o exercício é prescrito com $50 \%$ da carga de 1-RM e é realizado até a fadiga, esse exercício deve ser considerado máximo. Esse é um aspecto importante, principalmente para a prescrição do treinamento com pesos para grupos especiais, em que geralmente utiliza-se uma menor intensidade relativa de 1-RM na tentativa de atenuar os riscos à saúde, de forma inversa com o que ocorre no exercício intenso.

Adicionalmente, em virtude dos rápidos aumentos de força observados, sobretudo, nas primeiras semanas de treinamento com pesos, a carga de treinamento pode ser rapidamente subestimada quando a prescrição das cargas é baseada em percentuais de 1-RM, o que pode resultar em queda importante na intensidade do treinamento quando os testes de 1-RM não são aplicados com grande freqüência. Vale ressaltar, também, que testes de 1-RM não são específicos às exigências do treinamento para hipertrofia ou resistência muscular, uma vez que as exigências metabólicas em testes de 1-RM (sistema ATPCP) são diferentes daquelas observadas em exercícios com repetições múltiplas (sistema glicolítico e/ou oxidativo).

Considerando que para obtenção de medidas acuradas no teste de 1-RM são necessários alguns importantes cuidados metodológicos, apresentaremos a seguir as principais recomendações práticas para a aplicação deste teste.

\section{Recomendações práticas para a realização de testes de 1-RM}

Embora muitas dúvidas sobre a aplicação de testes de 1-RM já tenham sido desvendadas ao longo dos últimos anos por meio de diversas publicações, muitos aspectos que envolvem, sobretudo, a aplicação deste teste ainda merecem ser investigados de maneira mais consistente. Independente disso, algumas recomendações básicas podem ser extremamente relevantes, tanto para o avaliado quanto para os avaliadores envolvidos com a aplicação de testes de 1-RM, a saber:

a) Como em todo o teste que envolve esforço físico é necessário que o avaliado inicie a sessão de testagem, descansado e bem alimentado. Embora informações específicas a esse respeito não estejam disponíveis na literatura até 0 momento, acredita-se que 24 a $72 \mathrm{~h}$ de repouso, sem esforço extenuante seja suficiente;

b) Antes da aplicação do teste de 1-RM é necessário que o avaliado consiga executar com qualidade 0 gesto motor exigido, nas fases concêntrica e excêntrica do movimento. Assim, algumas sessões para familiarização com o equipamento a ser utilizado e aprendizagem da tarefa específica a ser exigida são necessárias. $O$ número de sessões é variável, dependendo, particularmente, do nível de aptidão física do avaliado, da complexidade do exercício (os multiarticulares são mais complexos do que os monoarticulares) e da sua experiência prévia com o exercício a ser testado ou, ainda, com a prática de exercícios com pesos;

c) Após o período de aprendizagem do movimento, algumas sessões de testes de 1-RM são necessárias para a estabilização da carga máxima e, consequentemente, para um registro adequado da força máxima. Duas a quatro sessões de familiarização especificamente com o teste de 1-RM a ser utilizado devem ser aplicadas, principalmente, em idosos, crianças, mulheres e indivíduos sem experiência em treinamento com pesos, em intervalos de 48 a 72 h. Vale destacar que o tempo necessário de intervalo entre as sessões, também necessita ser mais amplamente investigado. Não se pode desprezar que no caso da utilização de testes de 1-RM em praticantes de treinamento com pesos, - excesso de sessões de familiarização pode desmotivar o praticante e atrasar o início do programa de exercícios com pesos, uma vez que os testes de 1-RM não devem ser aplicados em dias de treinamento, principalmente, pelo desgaste gerado e pela excessiva duração do protocolo de testagem, sobretudo, se forem testados vários exercícios; 
d) Por se tratar de esforço máximo o avaliado deve apresentar um alto grau de motivação para alcançar o seu melhor desempenho físico. Como essa variavel é de difícil controle, o encorajamento verbal antes e durante a aplicação de testes de 1-RM é recomendado;

e) A utilização de alongamento prévio a aplicação de testes de 1-RM não é recomendada. Por outro lado, um aquecimento específico com um percentual da carga a ser testada inicialmente (em geral 40 a $60 \%$ ) tem sido utilizado com frequência na área de pesquisa. Entretanto, não existem estudos disponíveis que confirmem a necessidade ou não da aplicação desta conduta imediatamente antes de testes de 1-RM;

f) Cada sujeito deve ser avaliado em três a cinco tentativas, intervaladas por no mínimo três minutos entre elas para garantir um repouso adequado e ressíntese suficiente das reservas de ATP, de modo que não ocorra comprometimento energético para uma próxima tentativa. Uma ou duas tentativas podem não ser suficientes para um registro confiável da máxima carga a ser levantada em uma única ação muscular concêntrica, ao passo que o excesso de tentativas pode levar o indivíduo a uma fadiga residual e, consequente, perda de desempenho, subestimando a força máxima;

g) É recomendado que o teste não seja realizado em ambiente quente e úmido e que o avaliado esteja euhidratado.

\section{Considerações Finais}

As informações apresentadas neste estudo sugerem que: 1) $O$ teste de 1-RM apresenta segurança tanto ortopédica como cardiovascular; 2) Dependendo da população é importante que sejam realizadas múltiplas sessões de testes, em dias não consecutivos, até a estabilização da carga, para obtenção da medida acurada da força máxima; 3) Diversos fatores, tais como a realização de aquecimento ou alongamento previamente ao teste de 1-RM, o intervalo de recuperação entre as tentativas, a temperatura ambiente e a desidratação podem interferir nos resultados de 1-RM, atenuando ou maximizando 0 desempenho no teste; e 4) O teste de 1-RM parece ter boa aplicabilidade para avaliar as modificações na força decorrentes de programas de intervenção e para a avaliação da aptidão física em diferentes populações, contudo, a sua aplicação para a prescrição de programas de treinamento de força parece limitada.

\section{Referências}

ABERNETHY, P. J.; JURIMAE, J. Cross-sectional and longitudinal uses of isoinertial, isometric, and isokinetic dynamometry. Medicine and Science in Sports and Exercise, Hagerstown, v. 28, n. 9, p. 1180-1187, 1996.

\section{AMERICAN COLLEGE OF SPORTS MEDICINE (ACSM). ACSM's guidelines for exercise testing and prescription. Philadelphia: Lippincott, Williams and Wilkins, 2006.}

ASTORINO, T. A.; ROHMANN, R. L.; FIRTH, K. Effect of caffeine ingestion on one-repetition maximum muscular strength. European Journal of Applied Physiology, Berlin, v. 102, n. 2, p. 127-132, 2008. doi: <http://dx.doi.org/10.1007/s00421-007-0557-x>

BALADY, G. J.; BERRA, K. A.; GOLDING, L. A.; GORDON, N. F.; MAHLER, D. A.; MYERS, J. N.; SHELDAHL, L. M. ACSM's Guidelines for exercise testing and prescription. Philadelphia: Lippincott Williams \& Wilkins, 2000.

BARNARD, K. L.; ADAMS, K. J.; SWANK, A. M.; MANN, E.; DENNY, D. M. Injuries and muscle soreness during the one repetition maximum assessment in a cardiac rehabilitation population. Journal of Cardiopulmonary Rehabilitation, Philadelphia, v. 19, n. 1, p. 52-58, 1999.

BECK, T. W.; HOUSH, T. J.; SCHMIDT, R. J.; JOHNSON, G. O.; HOUSH, D. J.; COBURN, J. W.; MALEK, M. H. The acute effects of a caffeinecontaining supplement on strength, muscular endurance, and anaerobic capabilities. Journal of Strength and Conditioning Research, Champaign, v. 20, n. 3, p. 506-510, 2006.

BENTON, M. J.; SWAN, P. D.; PETERSON, M. D. Evaluation of multiple one repetition maximum strength trials in untrained women. Journal of Strength and Conditioning Research, Champaign, v. 23, n. 5, p. 1503-1507, 2009. doi: <http://dx.doi.org/10.1519/JSC.0b013e3181b338b 3>

CHAGAS, M. H.; BARBOSA, J. R. M.; LIMA, F. V. Comparação do número máximo de repetições realizadas a 40 e $80 \%$ de uma repetição máxima em dois diferentes exercícios na musculação entre os gêneros masculino e feminino. Revista Brasileira de Educação Física e Esporte, São Paulo, v. 19, n. 1, p. 5-12, 2005.

CRONIN, J. B.; HENDERSON, M. E. Maximal strength and power assessment in novice weight 
trainers. Journal of Strength and Conditioning

Research, Champaign, v. 18, n. 1, p. 48-52, 2004.

DE VOS, N. J.; SINGH, N. A.; ROSS, D. A.; STAVRINOS, T. M.; ORR, R.; FIATARONESINGH, M. A. Continuous hemodynamic response to maximal dynamic strength testing in older adults. Archives of Physical Medicine and Rehabilitation, Chicago, v. 89, n. 2, p. 343-350, 2008. doi:

<http://dx.doi.org/10.1016/j.apmr.2007.08.130>

FLECK, S. J.; DEAN, L. S. Resistance-training experience and the pressor response during resistance exercise. Journal of Applied

Physiology, Bethesda, v. 63, n. 1, p. 116-120, 1987.

GOMIDES, R. S.; COSTA, L. A.; SOUZA, D. R.; QUEIROZ, A. C.; FERNANDES, J. R.; ORTEGA, K. C.; MION JUNIOR, D.; TINUCCI, T.; FORJAZ, C. L. M. Atenolol blunts blood pressure increase during dynamic resistance exercise in hypertensives. British Journal of Clinical Pharmacology, London, v. 70, n. 5, p. 664-673, 2010. doi: <http://dx.doi.org/10.1111/j.13652125.2010.03742>

GURJÃO, A. L. D.; CYRINO, E. S.; CALDEIRA, L. F. S.; NAKAMURA, F. Y.; OLIVEIRA, A. R.; SALVADOR, E. P.; RITTI-DIAS, R. M. Variação da força muscular em testes repetitivos de 1-RM em crianças pré-púberes. Revista Brasileira de Medicina do Esporte, Niterói, v. 11, n. 6, p. 319324, 2005. doi: <http://dx.doi.org/10.1590/S151786922005000600003>

HEDLEY, A. M.; CLIMSTEIN, M.; HANSEN, R. The effects of acute heat exposure on muscular strength, muscular endurance, and muscular power in the euhydrated athlete. Journal of Strength and Conditioning Research, Champaign, v. 16, n. 3, p. 353-358, 2002.

KAELIN, M. E.; SWANK, A. M.; ADAMS, K. J.; BARNARD, K. L.; BERNING, J. M.; GREEN, A. Cardiopulmonary responses, muscle soreness, and injury during the one repetition maximum assessment in pulmonary rehabilitation patients. Journal of Cardiopulmonary Rehabilitation, Philadelphia, v. 19, n. 6, p. 366-372, 1999.

KOKKONEN, J.; NELSON, A. G.; CORNWELL, A Acute muscle stretching inhibits maximal strength performance. Research Quarterly for Exercise and Sport, Washington, v. 69, n. 4, p. 411-415, 1998.

LEVINGER, I.; GOODMAN, C.; HARE, D. L.; JERUMS, G.; TOIA, D.; SELIG, S. The reliability of the 1RM strength test for untrained middle-aged individuals. Journal of Science and Medicine in Sport, Belconnen, v. 12, n. 2, p. 310-316, 2009. doi: <http://dx.doi.org/10.1016/j.jsams.2007.10.007>

MACDOUGALL, J. D.; TUXEN, D.; SALE, D. G.; MOROZ, J. R.; SUTTON, J.R. Arterial blood pressure response to heavy resistance exercise. Journal of Applied Physiology, Bethesda, v. 58, n. 3, p. 785-790, 1985.

MATUSZAK, M. E.; FRY, A. C.; WEISS, L. W.; IRELAND, T. R.; MCKNIGHT, M. M. Effect of rest interval length on repeated 1 repetition maximum back squats. Journal of Strength and

Conditioning Research, Champaign, v. 17, n. 4, p. $634-637,2003$.

MURPHY, A. J.; WILSON, G. J. The ability of tests of muscular function to reflect traininginduced changes in performance. Journal of Sports Sciences, London, v. 15, n. 2, p. 191-200, 1997.

NASCIMENTO, M. A.; JANUÁRIO, R. B. S.; GERAGE, A. M.; MAYHEW, J. L.; PINA, F. L. C..; CYRINO, E. S. Familiarization and reliability of 1RM strength testing in older women. Journal of Strength and Conditioning Research, Champaign, 2012. [Epub ahead of print]. doi: $<$ http://dx.doi.org/10.1519/JSC.0b013e318271731 8>

NELSON, A. G.; KOKKONEN, J. Acute ballistic muscle stretching inhibits maximal strength performance. Research Quarterly for Exercise and Sport, Washington, v. 72, n. 4, p. 415-419, 2001

NELSON, A. G.; KOKKONEN, J.; ELDREDGE, C. Strength inhibition following an acute stretch is not limited to novice stretchers. Research Quarterly for Exercise and Sport, Washington, v. 76, n. 4, p. 500-506, 2005.

NICHOLS, J. F.; OMIZO, D. K.; PETERSON, K. K.; NELSON, K. P. Efficacy of heavy-resistance training for active women over sixty: muscular strength, body composition, and program adherence. Journal of the American Geriatrics Society, New York, v. 41, n. 3, p. 205-210, 1993.

PHILLIPS, W. T.; BATTERHAM, A. M.; VALENZUELA, J. E.; BURKETT, L. N. Reliability of maximal strength testing in older adults.

Archives of Physical Medicine and

Rehabilitation, Chicago, v. 85, n. 2, p. 329-334, 2004. doi:

<http://dx.doi.org/10.1016/j.apmr.2003.05.010>

PLOUTZ-SNYDER, L. L.; GIAMIS, E. L. Orientation and familiarization to $1 \mathrm{RM}$ strength testing in old and young women. Journal of Strength and Conditioning Research, Champaign, v. 15, n. 4, p. 519-523, 2001. 
POLLOCK, M. L.; CARROLL, J. F.; GRAVES, J. E.; LEGGETT, S. H.; BRAITH, R. W.; LIMACHER, M.; HAGBERG, J. M. Injuries and adherence to walk/jog and resistance training programs in the elderly. Medicine and Science in Sports and Exercise, Hagerstown, v. 23, n. 10, p. 1194-1200, 1991.

RITTI-DIAS, R. M.; CYRINO, E. S.; SALVADOR, E. P.; CALDEIRA, L. F. S.; NAKAMURA, F. Y.; PAPST, R. R.; BRUNA, N.; GURJÃO, A. L. D. Influência do processo de familiarização para avaliação da força muscular em testes de 1-RM. Revista Brasileira de Medicina do Esporte, Niterói, v. 11, n. 1, p. 34-38, 2005. doi: $<$ http://dx.doi.org/10.1590/S151786922005000100004>

RITTI-DIAS, R. M.; AVELAR, A.; SALVADOR, E. P.; CYRINO, E. S. Familiarização ao teste de 1RM em mulheres com experiência prévia em treinamento com pesos. Revista da Educação Física/UEM, Maringá, v. 20, n. 3, p. 423-429, 2009. doi:

<http://dx.doi.org/10.4025/reveducfis.v20i3.5941>

RITTI-DIAS, R. M.; AVELAR, A.; SALVADOR, E. P.; CYRINO, E. S. Influence of previous experience on resistance training on reliability of one-repetition maximum test. Journal of Strength and Conditioning Research, Champaign, v. 25, n. 5, p. 1418-1422, 2011. doi: <http://dx.doi.org/ 10.1519/JSC.0b013e3181d67c4b>

SALEM, G. J.; WANG, M.; SIGWARD, S. Measuring lower extremity strength in older adults: the stability of isokinetic versus 1RM measures. Journal of Aging and Physical Activity, Champaign, v. 10, n. 4, p. 489-503, 2002.

SALVADOR, E. P.; CYRINO, E. S.; GURJÃO, A. L. D.; RITTI-DIAS, R. M.; NAKAMURA, F. Y.; OLIVEIRA, A. R. Comparação entre o desempenho motor de homens e mulheres em séries múltiplas de exercícios com pesos. Revista Brasileira de Medicina do Esporte, Niterói, v. 11, n. 5, p. 257-261, 2005. doi: <http://dx.doi.org/10.1590/S151786922005000500002>

SCHILLER, B. C.; CASAS, Y. G.; TRACY, B. L.; DESOUZA, C. A.; SEALS, D. R. Age-related declines in knee extensor strength and physical performance in healthy Hispanic and Caucasian women. The Journals of Gerontology. Series A, Biological Sciences and Medical Sciences, Washington, v. 55, n. 12, p. B563-9, 2000. doi: <http://dx.doi.org/10.1093/gerona/55.12.B563>

SCHOFFSTALL, J. E.; BRANCH, J. D.; LEUTHOLTZ, B. C.; SWAIN, D. E. Effects of dehydration and rehydration on the one-repetition maximum bench press of weight-trained males. Journal of Strength and Conditioning Research, Champaign, v. 15, n. 1, p. 102-108, 2001.

SHAW, C. E.; MCCULLY, K. K.; POSNER, J. D. Injuries during the one repetition maximum assessment in the elderly. Journal of Cardiopulmonary Rehabilitation, Philadelphia, v. 15, n. 4, p. 283-287, 1995.

SIMÃO, R.; GIACOMINI, M. B.; DORNELLES, T. S.; MARRAMOM, M. G. F.; VIVEIROS, L. E. Influência do aquecimento específico e da flexibilidade no teste de 1RM. Revista Brasileira de Fisiologia do Exercício, Rio de Janeiro, v. 2, n. 2, p. 134-140, 2003.

SILVA-BATISTA, C.; TRICOLI, V. A. A.; LAURENTINO, G. C.; BATISTA, M. A. B.; OKUNO, N. M.; UGRINOWITSCH, C. Efeito da familiarização na estabilização dos valores de 1RM para homens e mulheres. Motriz, Rio Claro, v. 17 , n. 4, p. $610-617,2011$. doi: <http://dx.doi.org/10.1590/S198065742011000400005>

SIMÃO, R.; CÁCERES, M. S.; BÜRGER, F.; KOVALCZYK, L.; LEMOS, A. Teste de 1RM e prescrição de exercícios resistidos. Arquivos em Movimento, Rio de Janeiro, v. 2, n. 1, p. 55-63, 2006.

SOARES-CALDEIRA, L. F.; RITTI-DIAS, R. M.; OKUNO, N. M.; CYRINO, E. S.; GURJÃO, A. L.; PLOUTZ-SNYDER, L. L. Familiarization indexes in sessions of 1-RM tests in adult women. Journal of Strength and Conditioning Research, Champaign, v. 23, n. 7, p. 2039-2045, 2009. doi: <http://dx.doi.org/10.1519/JSC.0b013e3181b3e15 8>

TAAFFE, D. R.; PRUITT, L.; LEWIS, B.; MARCUS, R. Dynamic muscle strength as a predictor of bone mineral density in elderly women. The Journal of Sports Medicine and Physical Fitness, Torino, v. 35, n. 2, p. 136-142, 1995.

TIGGEMANN, C. L.; GUEDES, M. G.;

BGEGINSKI, R.; PINTO, R. S.; KRUEL, L. F. M. The reliability of the one maximum repetition in sedentary, active and strength-trained subjects. Motriz, Rio Claro, v. 17, n. 4, p. 700-707, 2011. doi: <http://dx.doi.org/10.1590/S198065742011000400015>

TRICOLI, V.; PAULO, A. C. Efeito agudo dos exercícios de alongamento sobre o desempenho de força máxima. Revista Brasileira de Atividade Física e Saúde, Londrina, v. 7, n. 1, p. 6-13, 2002. 
VERDIJK, L. B.; LOON, L. V.; MEIJER, K.;

SAVELBERG, H. H. C. M. One-repetition maximum strength test represents a valid means to assess leg strength in vivo in humans. Journal of Sports Sciences, London, v. 27, n. 1, p. 59-68, 2009. doi:

<http://dx.doi.org/10.1080/02640410802428089>

WEIR, J. P.; WAGNER, L. L.; HOUSH, T. J. The effect of rest interval length on repeated maximal bench press. Journal of Strength and

Conditioning Research, Champaign, v. 8, n. 1, p. 58-60, 1994.

WERBER-ZION, G.; GOLDHAMMER, E.; SHAAR, A.; POLLOCK, M. L. Left ventricular function during strength testing and resistance exercise in patients with left ventricular dysfunction. Journal of Cardiopulmonary Rehabilitation, Philadelphia, v. 24, n. 2, p. 100-109, 2004.

Agradecimentos: Os autores agradecem ao Conselho Nacional de Desenvolvimento Científico e Tecnológico (CNPq) pela bolsa de produtividade em pesquisa (E.S.C. e R.M.R.D.) e a Coordenação de Aperfeiçoamento de Pessoal de Nível Superior (CAPES) pelas bolsas de mestrado (A.L.M. e D.R.P.S.) e doutorado (A.A. e E.P.S.) outorgadas.

\section{Endereço:}

Edilson Serpeloni Cyrino

Universidade Estadual de Londrina

Rod. Celso Garcia Cid, km 380

Londrina PR Brasil

86057-970

Telefone/Fax: (43) 3371-4772

e-mail: emcyrino@uel.br

Recebido em:07 de novembro de 2011.

Aceito em: 18 de fevereiro de 2013.

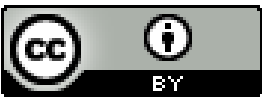

Motriz. Revista de Educação Física. UNESP, Rio Claro, SP, Brasil - elSSN: 1980-6574 - está licenciada sob Creative Commons - Atribuição 3.0 